\title{
Physics of multiphase microflows and microdroplets
}

\author{
Yonghao Zhang and Haihu Liu \\ Department of Mechanical Engineering, University of Strathclyde, Glasgow, G1 1XJ, UK
}

Multiphase microfluidic applications are very broad, ranging from DNA analysis such as PCR in droplets to chemical synthesis (Huebner et al. 2008). Optimal design and operation of such systems need insightful understanding of fundamental multiphase flow physics at microscale. In this chapter, we will select to discuss some basic flow physics of multiphase microdropets. The important dimensionless parameters relating to droplet dynamics will be elaborated. We will use droplet generation processes as examples to explain rich flow physics involved in microdroplet dynamics.

\section{Surface tension}

In comparison with single phase microfluidic flows, surface tension (also called interfacial tension) which plays a central role in dynamical behaviour of multiphase microdroplets. Over two centuries ago, Benjamin Franklin experimentally studied the effect of an insoluble fatty acid oil on the surface of water (Franklin et al. (1774)), which probably is the first time that the phenomenon of surface tension was given a scientific explanation.

For simplicity, we first consider a droplet in a carrier gas phase to explain surface tension. A liquid/gas interface will be presented, as shown in Figure 1, where fluid molecules will interact with each other. A molecule in the bulk liquid is attracted by all neighbouring molecules from all directions, so any attraction by another molecule from one direction is always balanced by another molecule from the opposite direction. Meanwhile, a molecule on the interface is in a different situation. It is attracted inward and to the side but no sufficient outward attraction to balance the inward attraction due to smaller amount of molecules outside in the gas. The consequence is that the attraction on an interface molecule is not balanced that induces the surface to contract and leads to surface tension.

The term surface tension can also be used interchangeably with surface free energy. Since the energy of a molecule at surface is higher than that of a molecule in bulk liquid, work needs to be done to move a molecule to surface from bulk liquid. The free energy of the system therefore increases. According to the thermodynamic principle, the free energy of the system always tends to a minimum. Therefore, the interface surface will tend to contract, forming least possible surface area. 


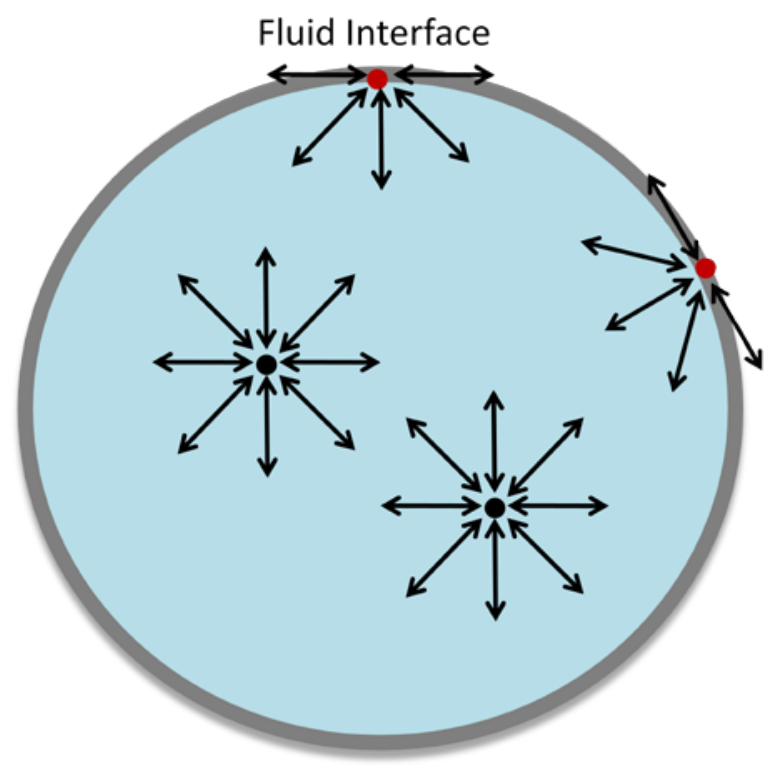

Figure 1. Illustration of inter-molecule interactions in the bulk and interface for a liquid droplet in gas.

The surface tension, represented by the symbol $\sigma$, can therefore be defined as a force per unit length or a surface free energy per unit area. Typical values at $20^{\circ} \mathrm{C}$ for water-air, ethanol-air, and mercury-air are 72.94 $\mathrm{mN} / \mathrm{m}, 22.27 \mathrm{mN} / \mathrm{m}$, and $487 \mathrm{mN} / \mathrm{m}$, respectively. Surface tension depends on temperature, and usually decreases as the liquid temperature increases. It can also be altered by surface-active materials, i.e. , surfactants, which form a monolayer at the interface. Due to large surface-to-volume ratio of microdroplets, surface tension often plays a dominant role in the determination of droplet behaviour.

\section{Young Laplace equation}

For a liquid droplet in another immiscible fluid, e.g. water droplet in air or oil, the pressure inside the droplet will normally be different from the outside pressure, because the surface tension leads to so called capillary pressure across the interface. For a stationary droplet in a rest surrounding immiscible fluid, i.e. the tangential stress is absent, the capillary pressure can be described by the Young Laplace equation as

$\Delta P=\sigma\left(\frac{1}{R_{1}}+\frac{1}{R_{2}}\right)$,

where $\Delta P$ is the capillary pressure i.e. the pressure difference across the fluid interface, and $R_{1}$ and $R_{2}$ are the principal radii of curvature. The equation is named after Thomas Young (who first proposed the theory of surface tension in 1805) and Pierre-Simon Laplace (who gave the mathematical description in 1806). This Young Laplace equation has been widely used as a bench-mark test case for multiphase models.

\section{Marangoni effects}

When local temperature, solvent concentration or electric potential is not uniform along the interface, the surface tension is not constant, i.e. there is surface tension gradient along the interface. Consequently, the 
gradients in surface tension lead to forces which are called Marangoni stresses, appear along the interface. The mass transfer along an interface between two fluids due to surface tension gradient is called Marangoni effects. If the phenomenon is temperature induced, it is often called thermo-capillary effects. Although this phenomenon was first identified by James Thomson in 1855, it is named after Carlo Marangoni because he studied this phenomenon in details for his doctoral dissertation at the University of Pavia and published his results in 1865.

\section{Navier-Stokes equation and surface tension model}

The commonly used fluids in microfluidic applications are Newtonian fluids, i.e., the shear stress of the fluid is linearly proportional to the applied shear rate. For a Newtonian fluid not far away from thermodynamically equilibrium, the Navier-Stokes equations can describe fluid dynamical behaviour. The continuum equation which considers the conservation of mass is given by

$\frac{\partial \rho}{\partial t}+\nabla \cdot(\rho \boldsymbol{u})=0$,

where $\rho$ is the fluid density, $\boldsymbol{u}$ is the velocity, $t$ is time. As fluids usually move at low speed in microfluidic applications (a typical velocity is up to $1 \mathrm{~cm} / \mathrm{s}$ ), flow can be considered as incompressible. The above continuum equation can be reduced to

$\nabla \cdot \boldsymbol{u}=0$.

Note: incompressible flow does not necessary mean that fluid density is constant, which only holds for steady flow where flowfields do not evolve in time. The momentum equation which considers the momentum conservation is described by:

$\rho \frac{D \boldsymbol{u}}{D t}=-\nabla p+\eta \nabla^{2} \boldsymbol{u}+\rho \boldsymbol{g}$,

where $p$ is static pressure, $\eta$ is fluid viscosity, $\boldsymbol{g}$ is gravity.

The above continuum and momentum equations are called the Navier-Stokes equations for single phase fluid. When an interface is presented in two immiscible Newtonian fluids, the interface, separating these two fluids, can be treated as a boundary condition which imposes an additional interface stress on fluids. Therefore, to consider the effect of interfacial stress, the above momentum equation becomes

$\rho \frac{D \boldsymbol{u}}{D t}=-\nabla p+\eta \nabla^{2} \boldsymbol{u}-\boldsymbol{F}_{s}+\rho \boldsymbol{g}$,

where $\boldsymbol{F}_{s}$ is the interfacial stress forcing term. A commonly used model is the Brackbill's continuum surface force (CSF) model where this surface forcing term is treated as a body force (Brackbill et al. (1992)), i.e.

$\boldsymbol{F}_{s}=\sigma \kappa \nabla C$,

where $C$ is the volume fraction of the fluids at the interface, $\kappa$ is the curvature of the local interface. 


\section{Numerical methods}

Experimentally, it is often difficult to measure local flowfield including velocity, pressure, and temperature at microscale. Modelling and simulation offer an important complimentary means to understand droplet dynamics and optimize device design and operation. Several numerical methods have been developed to describe the complex evolution process of a multiphase system. These methods can be classified into two major categories: the interface tracking and the interface capturing (Hirt and Nichols (1981); Osher and Sethian (1988); Anderson and McFadden (1998); Tryggvason et al. (2001)). The interface tracking method is a sharp interface approach, in which the interfaces are assumed to be infinitely thin i.e. zero thickness. A set of governing equations are applied to each phase or component, and the interfacial conditions are used as boundary conditions. Through iterations, the velocity of the interface is determined, and the interface then moves to a new location ready for the next time step. In this manner, the computations continue, and the interface is exactly tracked. This approach can provide very accurate results for cases without severe topological changes, and it forms the foundation of the front tracking methods (see Unverdi and Tryggvason (1992)). However, such an approach encounters singularity problems when significant topological changes (e.g., breakup and coalescence of droplets) occur. In these situations, artificial treatments or ad hoc criteria are required. In addition, this approach requires a large number of grid points on the interface in order to accurately represent large deformation, so dynamical local mesh refinements are essential to improve computational efficiency. However, significant research effort is required to overcome the computational difficulties associated with dynamic re-meshing and parallel computing.

Contrary to the interface tracking approach, the interface-capturing method uses a continuous function (to be called "indicator function" thereafter) to distinguish different phases. This type of approach is able to deal with topological changes in a natural way. The indicator function is generally chosen as the volume fraction of one of the two phases/components, as in the volume of fluid (VOF) method (Hirt and Nichols (1981)), the signed distance to the interface, as in the level-set method (Osher and Sethian (1988)), or the density/mass fraction of one phase or component (also called order parameter), as in the phase-field models (Anderson and McFadden (1998)). In this class of approach, the same set of governing equations (Eqs. $(2,5)$ ) are used for fluid flows. The fixed Eulerian grids are usually used for simulation domains and the interfaces are implicitly captured by the indicator function (known as "interface capturing"). Since the interface capturing methods have been widely used for multiphase microfluidic flow simulations, we will briefly discuss these methods below.

\subsection{Volume of fluid method}


The VOF method uses the volume fraction of one fluid phase or component (denoted as $C$ ) to characterize the interfaces (here, we refer to a two immiscible fluids). In the bulk phase (i.e. a pure fluid), $C$ is equal to zero or unity; in multi-fluid computational cells, $0<C<1$. In general, the VOF method consists of three major steps: the interface reconstruction algorithm, which provides an explicit description of the interface in each multi-fluid cell based on the volume fractions at this time step; the advection algorithm, which calculates the distribution of $C$ at the next time step by solving an advection equation (Eq. (7)) using the reconstructed interface and the solved velocity field at the previous time step; and the interfacial tension force model, which takes account of interfacial tension effects at the interface. Two widely-used interface reconstruction methods are simple line interface calculation (SLIC) (Hirt and Nichols (1981)) and the piecewise linear interface calculation (PLIC) (Gueyffier et al. (1999)). In the SLIC method, the volume of fluid in each cell is treated as if its local interface is either a vertical or horizontal line. In the PLIC method, the local phase interface is determined by fitting a straight line in the cell that satisfies the volume of fluid criteria, and the orientation of the straight line is decided by the distribution of one of the fluids in the neighbouring cells. In addition to these geometrical re-construction schemes, there are some other numerical schemes to solve the transport equation of indicator function. For example, Yabe and Xiao (1995) used a smooth function to transform $C$ to avoid rapid change of $C$ at the interface, which does not need a computationally costly interface re-construction step.

The volume fraction function is purely advected by the velocity field, i.e., it obeys the transport equation:

$\frac{\partial C}{\partial t}+\boldsymbol{u} \cdot \nabla C=0$.

Generally, the effect of interfacial tension force is incorporated into the momentum equation (Eq. (5)) using the continuum surface force (CSF) model of Brackbill et al. (1992). The normal vector and the curvature of the interface are calculated from derivatives of this volume fraction function. The interfacial tension force is applied using these two computed quantities, and its magnitude is proportional to the interfacial tension $\sigma$ (see Eq. (6)). Therefore, in addition to be an indicator, the volume fraction function plays an important role in the enforcement of the interfacial tension effect. This method is so far most likely to be found in commercially available computational fluid dynamics (CFD) software.

\subsection{Level set method}

The level set method is firstly introduced by Osher and Seithian (1988). The basic idea is to use a smooth function (level set function, $\varphi$ ) defined in the whole solution domain to represent the interface. It is defined as a signed distance to the interface and is purely a geometrical variable. The advantage is that the level set function varies smoothly across the interface, which eliminates the discontinuity problem that occurs in the VOF method. The CSF model for interface tension force is also used in the level set method. Similar to the 
volume fraction function in the VOF method, the level set function used in the level set method is purely transported by the flow velocity field as

$\frac{\partial \varphi}{\partial t}+\boldsymbol{u} \cdot \nabla \varphi=0$.

In contrast to the volume fraction, it is just an indicator that has no physical meaning. Therefore, the level set function does not need to satisfy the conservation law. It only needs to consider differentiation of the convection term. However, the level set method requires a re-initialization procedure to restore the signed distance property when large topological changes occur around the interface (Sussman and Fatemi (1999)). This may violate the mass conservation for each phase or component.

\subsection{Phase-field method}

Phase-field method originates from the theory for near-critical fluids, in which the fluid system is fundamentally viewed as a whole and the indicator function (i.e. order parameter $\theta$ ) is associated with the free energy of the system based on the Cahn-Hilliard theory (Cahn and Hilliard (1958)). The order parameter is a conserved variable that varies continuously over thin interfacial layers and is mostly uniform in the bulk phases. In phase-field method, the interfacial region has its own physics. As the interface thickness becomes smaller and smaller in comparison with the droplet size, it can be mathematically proved that phase-field model approaches the original sharp interface equations (Anderson and McFadden (1998); Liu and Shen (2003)). The equation of fluid motion which is modified to account for the presence of thin layer of interface can be applied over the entire flow domain. For example, the Navier Stokes equations can be modified to include a pressure tensor accounting for the interfacial tension. The pressure tensor can be derived by the use of reversible thermodynamic arguments. The interfacial tension can be given in terms of the excess free energy which is distributed through a 3-dimensional layer rather than being defined on a 2-dimensional surface.

The order parameter is evolved by the Cahn-Hilliard equation,

$\frac{\partial \theta}{\partial t}+\boldsymbol{u} \cdot \nabla \theta=\nabla \cdot(M \nabla \phi)$,

where $M$ is mobility and $\phi$ is the chemical potential. In the phase-field method, the interface sharpness is automatically maintained by the anti-diffusive term without losing the continuity. The interface structure is preserved as the interface evolves, so that the method does not require additional efforts for interface reconstruction and re-initialization step as in the VOF and level set methods (Enright et al. (2002); Enright et al. (2005)). In addition, the smooth representation of the interface as a region with the finite thickness prevents the numerical difficulties caused by the interface singularities. Detailed discussion on some important numerical issues related to phase-field method can be found in Jacqmin (1999). Since the phasefield method resolves the interface structure, and the thermodynamics is built into the model, it includes 
rich physics which is not available in the VOF and level set methods. Consequently, it has some distinctive advantages, e.g., dynamic contact angle becomes a part of solution rather than prescribed value.

\section{Flow physics clarification: important dimensionless parameters}

As flow physics at microscale can be very different from the conventional scales, it is important to clarify physical phenomena occurring at small scales. Dimensionless numbers which evaluate the importance of these phenomena are useful for us to understand the underlying flow mechanisms of a flow system. Therefore, we discuss some important dimensionless numbers in this chapter.

\subsection{Reynolds number}

For fluid dynamics, the most widely used important dimensionless number is Reynolds number, Re, which compares inertial forces to viscous forces, i.e.

$\operatorname{Re}=\frac{\rho u L}{\eta}$,

where $L$ is the characteristic length of the flow system. Usually, Re is used to judge whether flow is laminar or turbulent. However, for microflows, the characteristic length is usually low, so the Reynolds number is small. This indicates that the inertial forces are relatively not important compared with the viscous forces. Typically, microfluidic devices use aqueous solutions which move at a speed between $1 \mu \mathrm{m} / \mathrm{s}$ to $1 \mathrm{~cm} / \mathrm{s}$. And the typical microchannels have height of 1-100 micron. Therefore, the Reynolds number is typically in the range of orders of $10^{-6}$ and 1 . So the flows are laminar and the inertial forces may be neglected. The conventional Navier-Stokes equation can therefore reduce to the Stokes equation, which is given by

$\rho \frac{\partial \boldsymbol{u}}{\partial t}=-\nabla p+\eta \nabla^{2} \boldsymbol{u}+\rho \boldsymbol{g}$.

Comparing with the Navier Stokes equation, the nonlinear term $\rho \boldsymbol{u} \cdot \nabla \boldsymbol{u}$ is gone in the right hand of the equation. Note: as conventional flow devices are usually operated at higher Re, the counter-part microfluidic devices should not be simply designed by scaling down the conventional devices.

\subsection{Capillary number}

While the most important dimensionless number for fluid dynamics, Re, is least interesting for microfluidics, the usually ignored interfacial tension in conventional free surface/interfacial flows become essential for micorfluidics. The corresponding dimensionless number is capillary number which compares surface tension forces with viscous forces.

$\mathrm{Ca}=\frac{\eta \boldsymbol{u}}{\sigma}$

where the viscosity of continuum phase is usually used.

\subsection{Bond number and Weber number}


The Bond number (Bo) (also known as Eötvös number (Eo)) is to evaluate buoyancy force against surface tension force,

Bo $=\frac{\Delta \rho \mathrm{g} L^{2}}{\sigma}$,

where $\Delta \rho$ is the density difference between two phases. It is an important parameter for describing droplet dynamical behaviour when the continuum carrier phase is gas. If we consider typical microfluidic water droplet in oil or oil droplet in water, the Bo number may not be essential as density difference between the immiscible liquid phases is small.

The Weber number (We), named after Moritz Weber (1871-1951), is regarded as a measure of the relative importance of the fluid's inertia in comparison with its surface tension,

$\mathrm{We}=\frac{\rho u^{2} L}{\sigma}$.

It is not an independent parameter which can be determined by Re and Ca, i.e. We $=\mathrm{Re}^{*} \mathrm{Ca}$. Weber number is usually not important for usually low speed microfluidic microdroplet applications.

\section{Droplet generation in microfluidic devices}

Generating uniform droplets is one important step of achieving microdroplet functionalities. Using pressure as driving force to generate droplets is one of the fastest and commonly used methods. Many microfluidic devices have been designed to apply pressure to generate uniform droplets, including geometry-dominated devices (Yasuno et al. (2004); Sugiura et al. (2004)), flow-focusing devices (Anna et al. (2003); Cubaud et al. (2005); Garstecki et al. (2005); Tan et al. (2008); Fu et al. (2009)) T-junctions (Thorsen et al. (2001); Nisisako et al. (2002); Guillot and Colin (2005); Garstecki et al. (2006); Graaf et al. (2006); De Menech et al. (2008); Christopher et al. (2008)) and co-flowing devices (Umbanhowar et al. (2000); Hua et al. (2007)). For device design optimization and operation, it is important to understand the underlying mechanisms of droplet generation processes in microchannels. In comparison with unbounded flows, the two-phase flow characteristics in microchannels is determined by not only flow conditions and fluids properties but also channel geometry. Here, we select two most popular device configurations - T-junctions and cross-junctions - and discuss droplet generation mechanisms in details.

\subsection{Droplet generation at T-junctions}

T-junctions are one of the most frequently used microfluidic geometries to produce immiscible fluid segments (plugs) and droplets. Although this approach has been widely used, the currently available information is still fragmented due to differences in channel dimensions, flow rates, fluid properties and surface materials. The research challenge still remains to fully understand underlying mechanisms of droplet formation processes that are influenced by capillary number, flow rate ratio, viscosity ratio, contact angle 
and channel geometrical configurations. Meanwhile, some important advances have been recently made in experimental and numerical studies. For example, a squeezing mechanism due to confined geometry in droplet formation process, which does not exist in an unbounded flow condition, has been identified by Garstecki et al. (2005). In the following sections, we will discuss state of the art of this research topic. The configuration of a typical T-junction is illustrated in Figure 2.

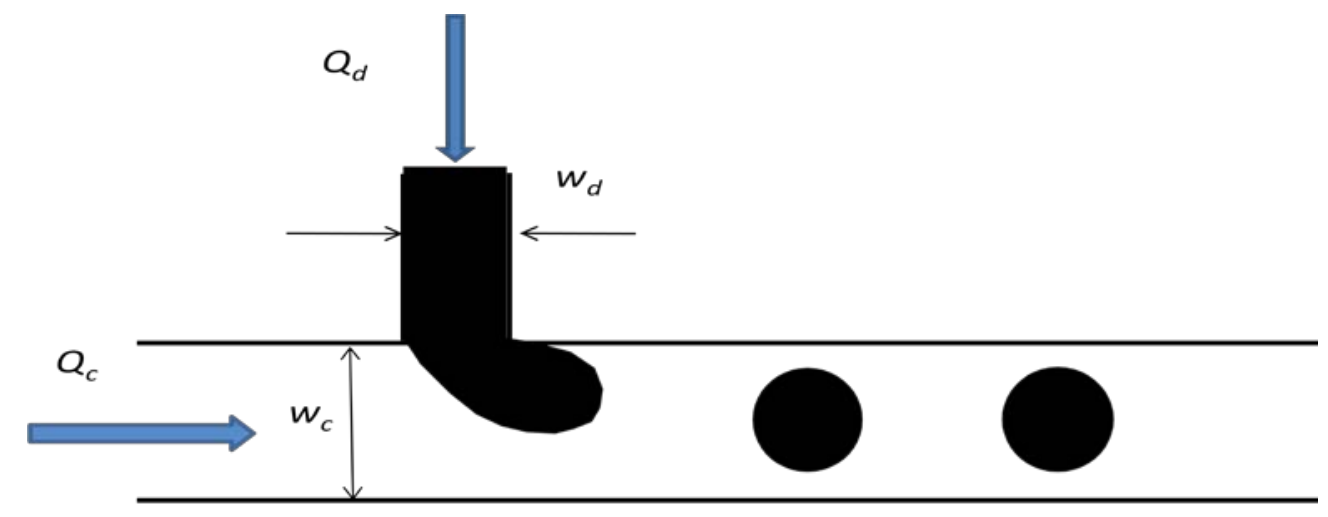

Figure 2 Droplet generation in a microfluidic T-junction with the disperse phase injected through the side channel and the carrier phase injected through the main channel. $Q_{c}$ and $Q_{d}$ are volume flow rate of the carrier and disperse phases respectively, while $w_{c}$ and $w_{d}$ are the width of the main and side channels.

\subsubsection{The flow regimes}

De Menech et al. (2008) identified three distinctive flow regimes: squeezing, dripping and jetting. As jetting occurs at very high flow rates or capillary number, this regime is not often utilized in microfluidic applications. The authors found in their computational study that in the squeezing regime, droplets (plugs) are generated in a way very different from unconfined cases. The breakup process is dominated by the buildup pressure in the upstream of an emerging droplet which blocks or partially blocks the main flow channel. Meanwhile, in the dripping regime, both buildup pressure and shear stress are important. This finding has been experimentally observed (e.g. Nisisako et al. (2002), Nisisako et al. (2004), England et al. (2010)). Figure 3 shows that plug fully blocks the main channel so that the bulidup pressure will pinch off the plug. The breakup point of plug is at the junction corner and capillary number is very small. Figure 4 shows that with larger capillary number, the droplet emerges out of the side channel will experience shear force from the carrier fluid and buildup pressure due to partial blockage of the main channel. The breakup point in this dripping regime is at the downstream of the main channel. 


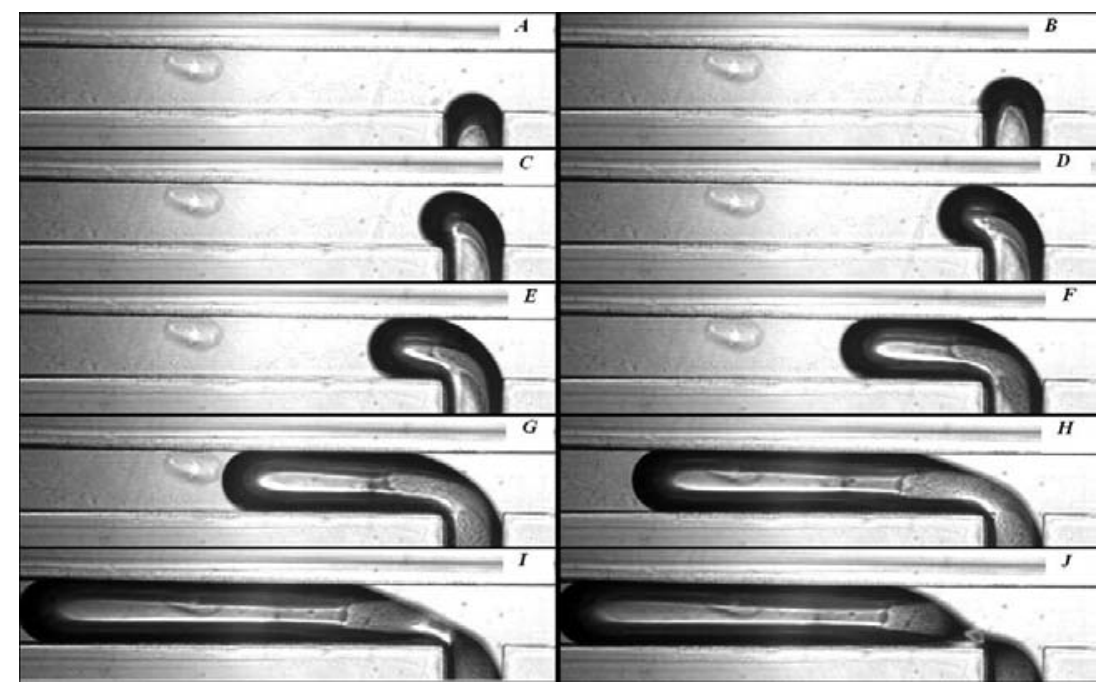

Figure 3: The droplet generation in the squeezing regime ( $\mathrm{A}$ to J) with $C a=0.0036$ and $Q=1.2$, where $\mathrm{Ca}$ is defined as Eq. (15) and $Q$ is the flow rate ratio $\left(Q_{d} / Q_{c}\right)$.

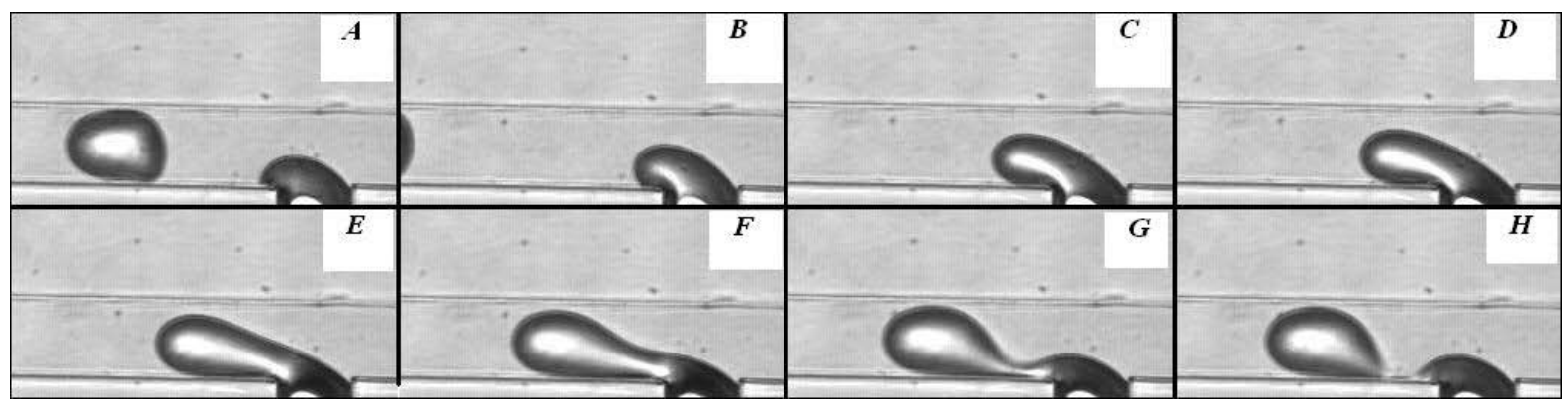

Figure 4: The droplet generation in the dripping regime (A to $\mathrm{H}$ ) with $C a=0.036$ and $Q=1.2$, where $\mathrm{Ca}$ is defined as Eq. (15) and $Q$ is the flow rate ratio $\left(Q_{d} / Q_{c}\right)$.

Here, we focus on droplet generation processes in the squeezing and dripping regimes. Flow behaviour in a microfluidic T-junction can be classified by a group of dimensionless parameters, which are commonly defined by the experimentally measurable variables e.g. the interfacial tension, the inlet volumetric flow rates $\left(Q_{c}\right.$ and $\left.Q_{d}\right)$ and viscosities $\left(\eta_{c}\right.$ and $\left.\eta_{d}\right)$ of the two fluids. For a typical microfluidic system, the Reynolds number is so small that the inertial effect can be neglected. The Bond number is also negligibly small due to the small density difference between two immiscible liquids. In contrast, the capillary number is the most important parameter in droplet generation processes, which can be defined by the average inlet velocity $u_{c}$ and the viscosity $\eta_{c}$ of the continuous phase, and the interfacial tension $\sigma$ as

$C a=\frac{\eta_{c} u_{c}}{\sigma}$ 


\subsubsection{Influence of the capillary number}

Fig.5 illustrates droplet formation process in the T-junction in the squeezing regime (a) and the dripping regime (b). The droplet emerges from the side channel and deforms before detachment, and the necking of the dispersed phase is initiated once the continuous phase fluid intrudes into the upstream side of the side channel. The intrusion of the continuous phase accentuates the influence of the contact line dynamics, which is thought to be indispensable for the droplet detachment. Fig.5 shows that the necking occurs right after the dispersed phase moves into the main channel when $\mathrm{Ca}$ is large (the dripping regime), while the plugs are formed when $\mathrm{Ca}$ is small (the squeezing regime). This is both confirmed in experimental and numerical studies (e.g. Nisisako et al. (2002), Nisisako et al. (2004), Liu and Zhang (2009), England et al. (2010)).
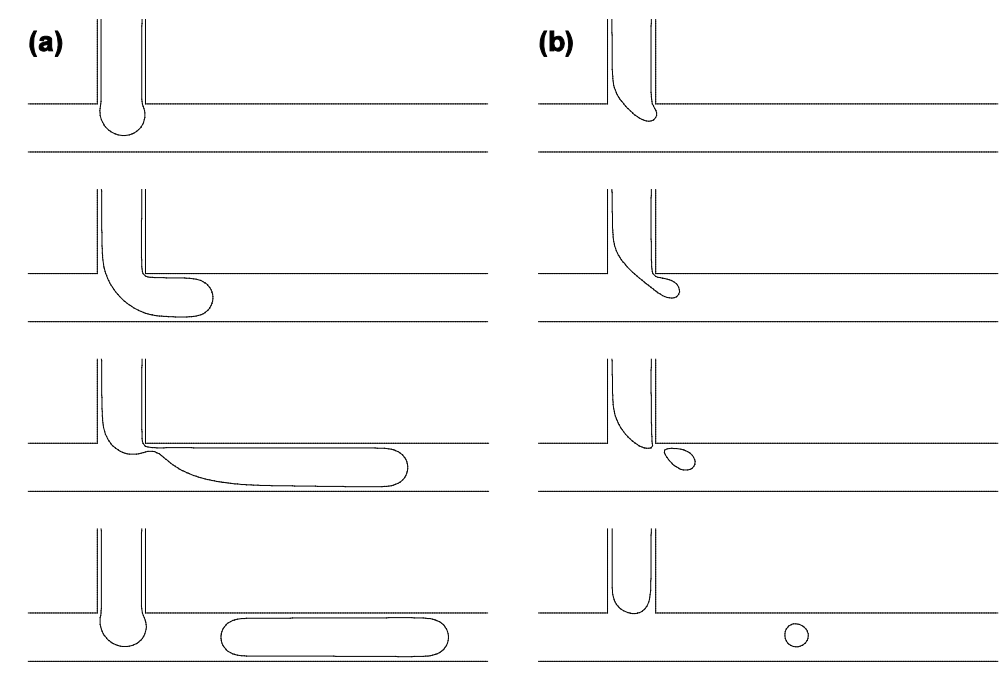

Figure 5 An illustration of droplet generation flow regimes in T-junction: a) squeezing regime; b) dripping regime. The figure is obtained from Liu and Zhang (2009) and copyright is awaited from Journal of Applied Physics.

Liu and Zhang (2009) showed that when the capillary number is low i.e. $\mathrm{Ca}=0.006$ in Fig. $6 a$, the incoming dispersed phase fluid tends to occupy the full width of the main channel, and the breakup occurs at the downstream side of T-junction corner. When the capillary number increases i.e. $\mathrm{Ca}=0.032$ and 0.056 in Figs. $6 \mathrm{~b}$ and $6 \mathrm{c}$, the dispersed phase fluid occupies only part of the main channel, and smaller droplets are formed. According to $\mathrm{Ca}$, two distinctive droplet generation regimes i.e. the squeezing and dripping regimes are identified. In the squeezing regime when $\mathrm{Ca}$ is small, the buildup of pressure at the upstream due to the obstruction of the main channel by the emerging droplet is responsible for the droplet "pinching off", while the viscous shear force becomes increasingly important in the dripping regime when Ca increases. 

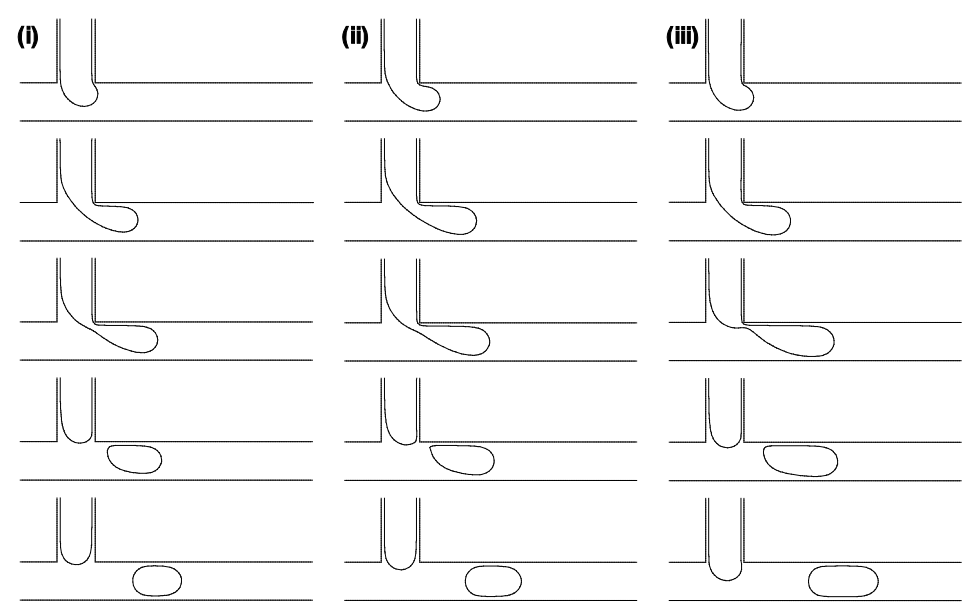

(a)
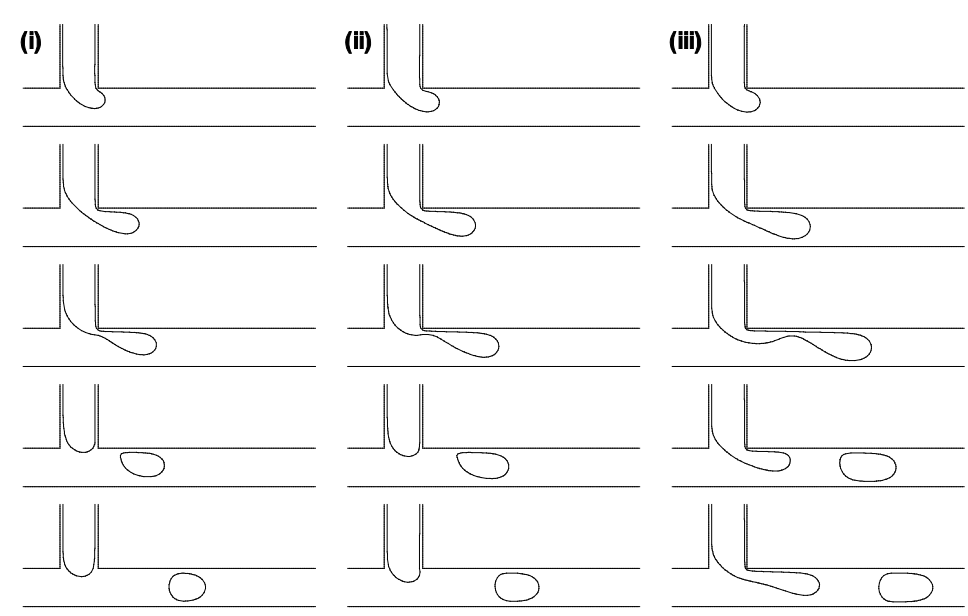

(b)
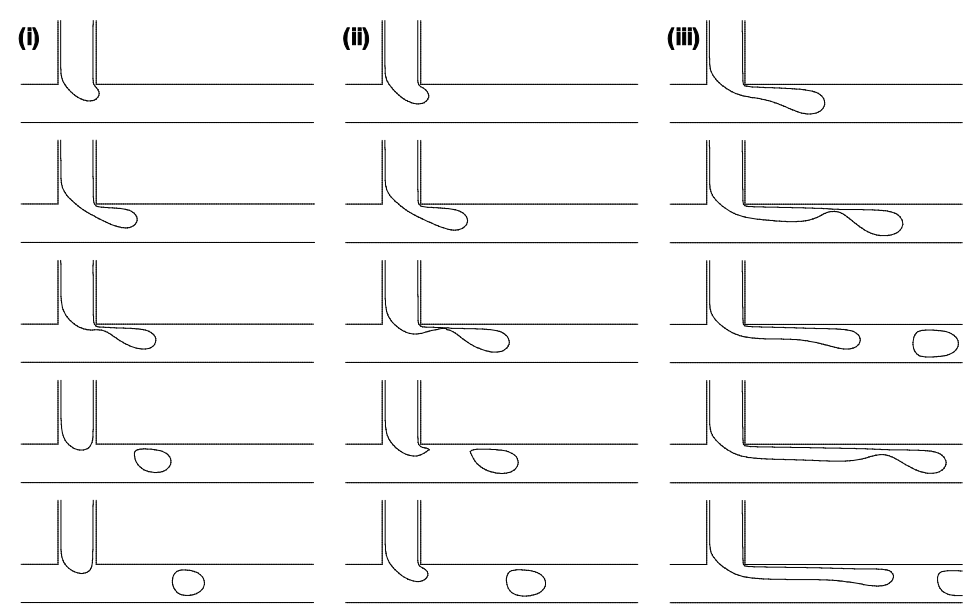

(c)

Figure 6 The effect of capillary number and flow rate ratio in droplet generation process, where $\mathrm{Ca}$ is (a) 0.06 , (b) 0.032 , and (c) 0.056 ; the flow rate ratio $Q$ is (i) $1 / 8$, (ii) $1 / 4$ and (iii) $1 / 2$. The figure is obtained from Liu and Zhang (2009) and copyright is awaited from Journal of Applied Physics. 
In both experimental and numerical studies, van der Graaf et al. $(2005,2006)$ found that the final droplet volume is a consequence of a two-stage droplet growth. Initially, the droplet grows to a critical volume $V_{c}$ until the forces exerted on the interface become balanced. Subsequently, the droplet continues to grow for a time $t_{n}$ for necking due to the continuous injection of the dispersed phase fluid. And the final droplet volume $V$ can be predicted by the scaling law below (van der Graaf et al. (2005)):

$V=V_{c}+t_{n} Q_{d}$

where $V_{c}$ depends only on $\mathrm{Ca}$ and the duration of necking $t_{n}$ and decreases as $\mathrm{Ca}$ increases. An empirical correction was proposed to improve the prediction of the droplet volume by van der Graaf et al. (2006):

$V=V_{c, r e f} C a^{m}+t_{n, r e f} C a^{n} Q_{d}$

where $V_{c, \text { ref }}$ and $t_{n, \text { ref }}$ are the reference values at $\mathrm{Ca}=1$ where the droplet detachment process is very fast, i.e. $t_{n} \rightarrow 0$; the exponents $m$ and $n$ depend on the device geometry, which were reported to be -0.75 (van der Graaf et al. (2006)).

\subsubsection{Influence of the flow rate ratio}

Apart from capillary number, flow rate ratio $Q\left(Q=Q_{d} / Q_{c}\right)$, plays an essential role in droplet generation processes. For small $Q$, the droplets are pinched off at the T-junction corner regardless of the capillary number. However, for larger $Q$, increasing Ca will force the detachment point to move from the corner to the downstream. Liu and Zhang (2009) showed in Fig. 6 that when Ca is fixed at 0.006 , varying $Q$ from $1 / 8$ to $1 / 2$ does not change the detachment point of the droplet. When $\mathrm{Ca}$ is increased to 0.032 and 0.056 , the detachment point will move from the T-junction corner to the downstream as $Q$ increases. In addition, the droplet detachment point gradually moves downstream until a stable jet is formed when Ca and $Q$ increase, which was also observed both numerically (De Menech et al. (2008); Liu and Zhang (2009)) and experimentally (Christopher et al. (2008)).

The droplet grows as the flow rate ratio increases but becomes smaller as the capillary number increases. In addition to the capillary number, flow rate ratio will affect the formed droplet size significantly. Fig. 6a shows that, in the squeezing regime, the flow rate ratio shows significant effect on the droplet size. In the dripping regime as $\mathrm{Ca}$ increases, the effect of the flow rate ratio interestingly diminishes, which was also recently reported by De Menech et al. (2008).

Many experimental studies were carried out in the squeezing regime so that the droplets filled the main channel and formed "plug-like" or "slug-like" shapes (Tice et al. (2003); Garstecki et al. (2006); Zheng et al. (2004)), where the viscous shear force may be ignored and the dominant force responsible for droplet breakup is the squeezing pressure caused by the channel obstruction. Garstecki et al. (2006) argued that the 
detachment begins once the emerging droplet fills the main channel and the droplet continues to grow during this time due to continuous injection of the dispersed phase fluid. Assuming that the neck squeezes at a rate proportional to the average velocity of the continuous phase fluid, and the plug fills at a rate proportional to $Q_{d}$, a scaling law for the final plug length was proposed:

$I / w_{c}=1+\alpha Q$,

where $\alpha$ is a constant of order one, whose value depends on the widths of both channels. It clearly shows the plug length depends only on Q. However, Liu and Zhang (2009) suggested that the droplet size also strongly depends on $\mathrm{Ca}$ in the squeezing regime, which is consistent with the experimental observations (e.g. Christopher et al. (2008)). Therefore, the role of capillary number needs to be reflected in the scaling law. Although the scaling law Eq. (19) does not capture the capillary number dependency, it can predict the droplet size under various flow rate ratios when $\mathrm{Ca}$ is fixed in the squeezing regime. When $\mathrm{Ca}$ is taken into account, the scaling law given by Eq. (18) should be used.

\subsubsection{Influence of viscosity ratio and contact angle}

As shown in Fig. 7, in the squeezing regime, the predicted droplet diameter is nearly independent of the viscosity ratio, $\lambda\left(\lambda=\eta_{d} / \eta_{c}\right)$, where the droplet formation is completely controlled by the capillary force and the squeezing pressure. In the dripping regime, the influence of viscosity ratio becomes more pronounced as Ca increases, where the large viscosity ratio leads to smaller droplet (De Menech et al. (2008). Liu and Zhang (2009)). However, it also shows that the influence of the viscosity ratio on the generated droplet diameter is not as significant as in the unbounded flow (Umbanhowar et al. (2000)), where the breakup of droplets is controlled by a competition between the viscous shear force and the capillary force. This indicates that the squeezing pressure caused by the confinement of geometry of a T-junction has to be taken into account even in the dripping regime.

Due to large surface to volume ratio, fluid/surface interaction will significantly affect the droplet dynamics in microchannels. The contact angle influences droplet shape, generation frequency, and detachment point. Liu and Zhang (2009) showed that the generated droplets become smaller when the contact angle increases. Interestingly, they also found that negligible viscosity ratio effect in the squeezing regime is only valid for more hydrophobic wetting conditions. 


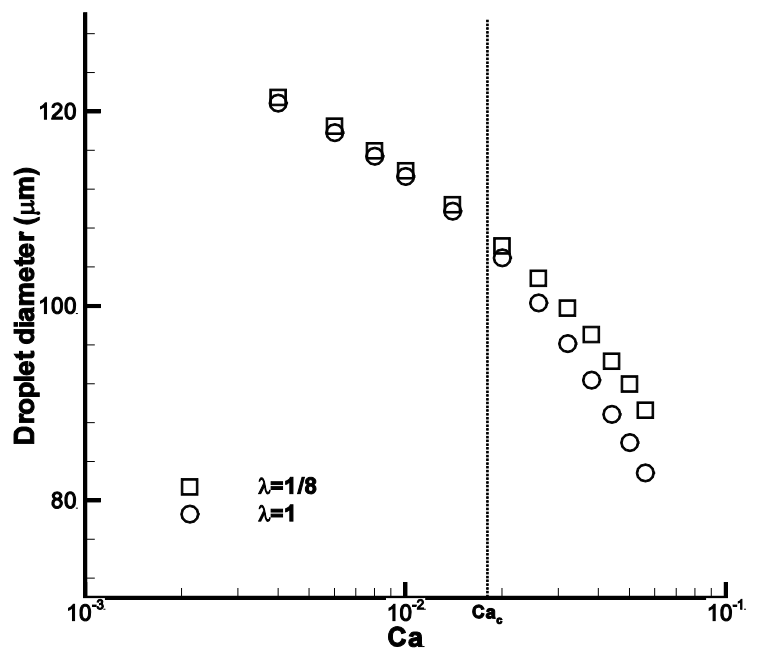

Figure 7 Influence of viscosity ratio on droplet size. The figure is obtained from Liu and Zhang (2009) and copyright is awaited from Journal of Applied Physics.

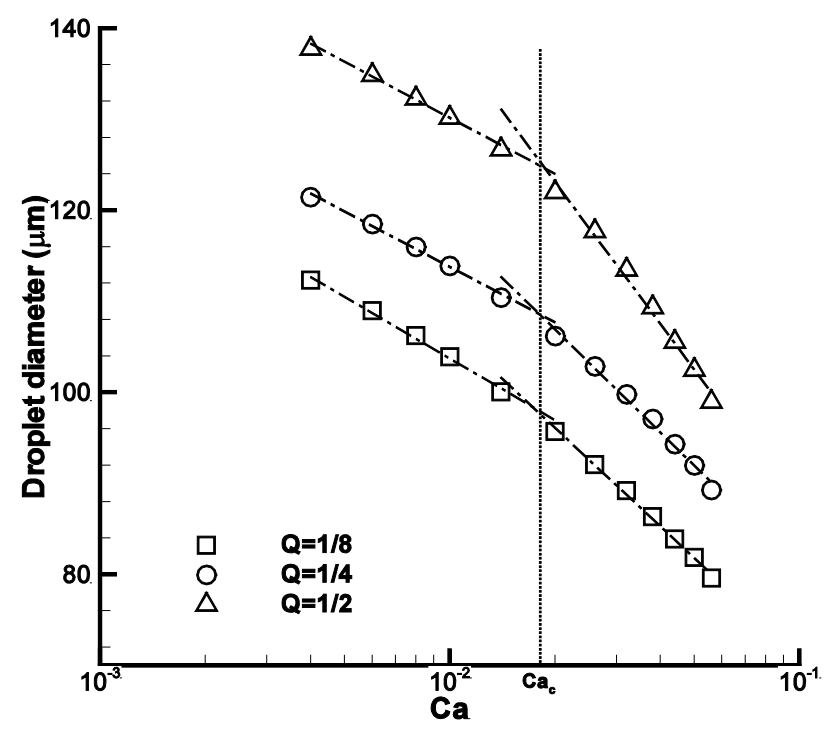

Figure 8 Squeezing-to-dripping flow regime transition. The figure is obtained from Liu and Zhang (2009) and copyright is awaited from Journal of Applied Physics.

\subsubsection{Regime change: Critical Capillary number}

Three flow regimes for droplet generation in T-junction i.e. squeezing, dripping and jetting have been identified. It is important to understand the factors that control regime transition especially squeezing-todripping transition which is most relevant to microfluidic microdroplet applications. The recent work has suggested that transition from squeezing to dripping regime depends on a critical capillary number. For 
example, De Menech et al. (2008), using the Navier-Stokes solver with a phase-field model, reported a critical capillary number of 0.015 . However, the recent experimental study by Christopher et al. (2008) did not observe the critical capillary number during the squeezing-to-dripping transition. Liu and Zhang (2009) noticed that the two regimes become difficult to distinguish as $Q$ decreases because the droplet detachment point is always close to the downstream corner of the T-junction at small $Q$. This may explain why Christopher et al. (2008) did not observe the critical Ca during the squeezing-to-dripping transition because they performed experiments at small viscosity ratio of 0.01 , where the droplet breakup always occurs at the downstream corner of the T-junction. According to Liu and Zhang (2009), there is a critical capillary number (see Fig. 8, $\mathrm{Ca}_{\mathrm{c}}=0.018$ ), which distinguishes the squeezing and dripping regimes. Furthermore, they showed that this critical capillary number is independent of the flow rate ratio, the viscosity ratio and contact angle. However, their work is based on 2D simulation results, so how channel depth will affect the critical capillary number remains to be investigated.

\subsection{Droplet generation in cross-junctions}

In comparison with droplet generation at T-junctions, droplet generation in a confined cross-junction is quite similar. The coupled factors which affect the droplet formation process at T-junction are also important, i.e. interfacial tension, wetting properties and confinement of flow channels, fluid flow rates and viscosities. In this section, we will highlight the difference between the droplet generation processes of cross and T-shaped junctions.
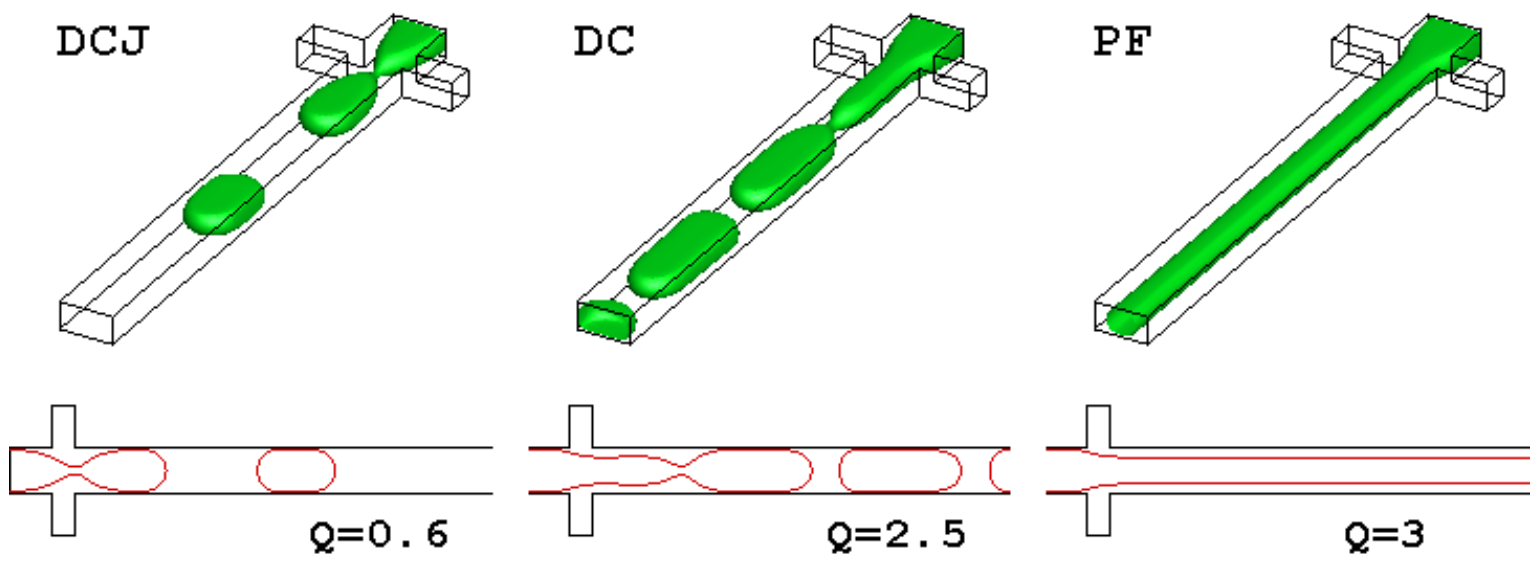

Figure 9 Typical three flow patterns at $\mathrm{Ca}=0.004$ and $\mathrm{Q}=0.6(\mathrm{DCJ}), 2.5(\mathrm{DC})$ and $3(\mathrm{PF})$. The figure is obtained from Liu and Zhang (2011) and copyright is awaited.

\subsubsection{Cross-junction flow patterns}

Similar to T-junctions as experimentally observed by Guillot and Colin (2005), there are also three typical flow patterns in droplet generation at low capillary number: the droplets are formed at the cross-junction 
(DCJ); at downstream of the cross-junction (DC), forming a thread that becomes unstable after a distance of laminar flow; the stable parallel flows (PF), where the three incoming streams co-flow in parallel to the downstream without pinching. The flow pattern transition will be affected by capillary number and flow rate ratio (see Fig. 9).

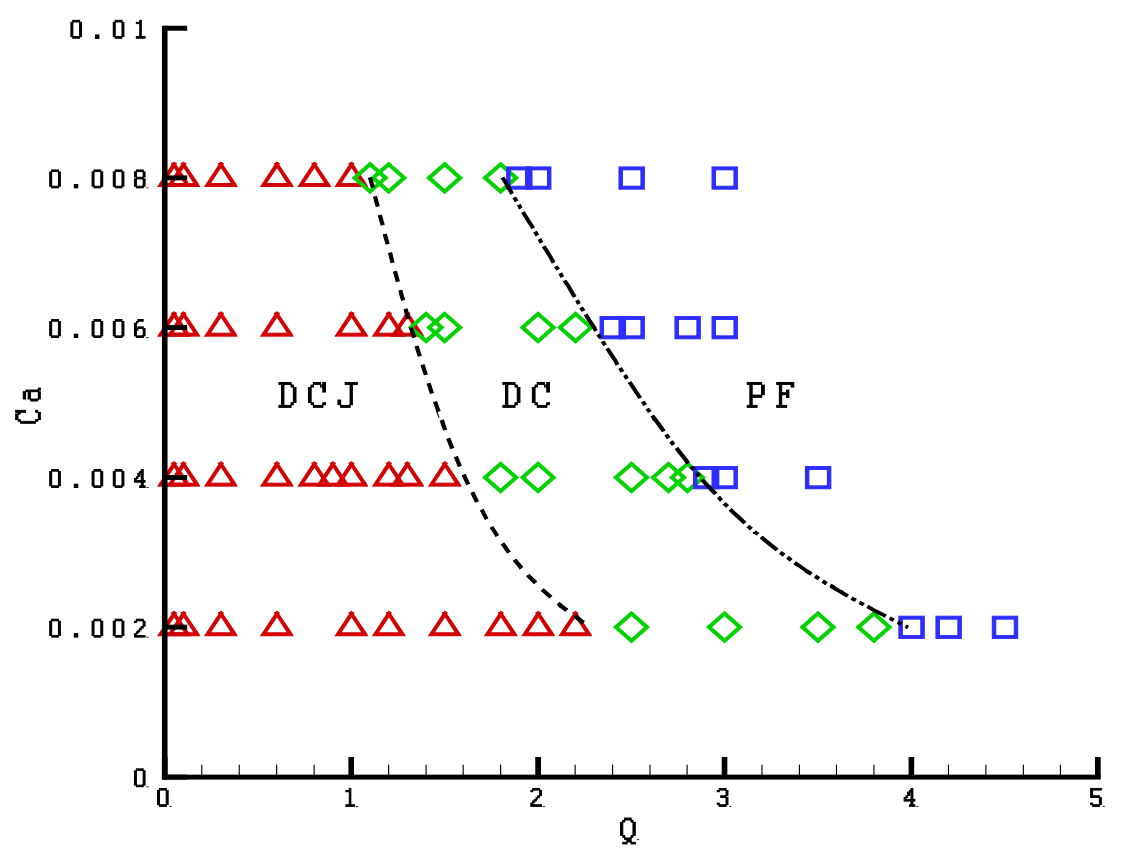

Figure 10 Droplet flow patterns as a function of flow rate ratio $Q$ and capillary number: Symbols indicate $\Delta$ : $D C J, \diamond: D C$, and $\square:$ PF. (the geometry configuration and viscosity ratio may also alter the flow pattern transition). The figure is obtained from Liu and Zhang (2011) and copyright is awaited.

\subsubsection{Scaling laws for droplet size}

On the basis of the experimental observation of plug formation at microfluidic T-junctions, Garstecki et al. (2006) argued that at low Ca the final length of a plug is contributed by two steps. First, the thread of the dispersed phase grows until it blocks the continuous phase liquid. At this moment the 'blocking length' of the plug is equal to $w_{c}$. Then the increased pressure in the continuous phase liquid begins to 'squeeze' the neck of dispersed thread. They proposed a scaling law to predict droplet size as given by Eq. (19). Recently, Xu et al. (2008) compared the experimental data from different authors and found that the 'blocking length' is not always equal to $w_{c}$, but is also dependent on the channel geometry. Therefore, the scaling law given by Eq.(19), is modified as

$\frac{L}{w_{c}}=\varepsilon+\omega Q$,

where $\varepsilon$ and $\omega$ are fitting constants that are mainly dependent on the channel geometry. 
Experimentally, in the DCJ, Tan et al. (2008) observed that the non-dimensional length of plugs $\left(L / w_{c}\right)$ exhibits a power-law dependence on the capillary number, i.e. $L / w_{c}=\mathrm{kCa}^{\mathrm{m}}$, which is independent of the flow rate ratio $Q$. Considering the influence of capillary number and flow rate ratio, Liu and Zhang (2011) proposed that the generated plug length (droplet diameter) can be predicted by

$\frac{L}{w_{c}}=(\varepsilon+\omega Q) \mathrm{Ca}^{m}$,

where $\varepsilon, \omega$ and $m$ are the fitting parameters that vary with the channel geometry. Based on their simulation results, Liu and Zhang (2011) confirm this scaling law and determine the coefficients ( $\varepsilon=0.551, \omega=0.277$ and $m=-0.292$ ). This is broadly consistent with the two-step model proposed by Graaf et al. (2005), which was general enough to describe the T-junction droplet formation (Steegmans et al. (2009)).

\section{Conclusion}

We do not aim to summarize complex multi-scale multi-physical droplet dynamics in a single chapter. In stead, we select the basic multiphase flow physics and pick droplet generation processes in microfluidic channels as examples to determine uniqueness of droplet dynamical behaviour in confined microchannels. We hope this chapter can be useful for readers new to flow physics which underpins microfluidic microdroplet technologies.

\section{References}

D. M. Anderson, G. B. McFadden, Diffuse-interface methods in fluid mechanics, Annu. Rev. Fluid Mech. 30 (1998) 139-165.

S. L. Anna, N. Bontoux, H. A. Stone, Formation of dispersions using "flow focusing" in microchannels, Appl. Phys. Lett. 82 (2003) 364-366.

J. U. Brackbill, D. B. Kothe, C. Zemach, A continuum method for modelling surface tension, J. Comput. Phys. 100 (2) (1992) 335-354.

J.W. Cahn, J. E. Hilliard, Free energy of a nonuniform system. I. Interfacial free energy, J. Chem. Phys. 28 (2) (1958) 258-267.

G. F. Christopher, N. N. Noharuddin, J. A. Taylor, S. L. Anna, Experimental observations of the squeezing-todripping transition in T-shaped microfluidic junctions, Phys. Rev. E 78 (2008) 036317.

T. Cubaud, M. Tatineni, X. Zhong, C.-M. Ho, Bubble dispenser in microfluidic devices, Phys. Rev. E 72 (2005) 037302.

M. De Menech, P. Garstecki, F. Jousse, H. A. Stone, Transition from squeezing to dripping in a microfluidic Tshaped junction, J. Fluid Mech. 595 (2008) 141-161.

P. England, H. H. Liu, Y. H. Zhang, S. Mohr, N. Goddard, P. Fielden, C. H. Wang, Experimental study of droplet formation at microfluidic t-junctions, Proceedings of the $2^{\text {nd }}$ European Conference on Microfluidics -

Microfluidics 2010 - Toulouse, December 8-10, 2010,paper No. 187. 
D. Enright, S. Marschner, R. Fedkiw, Animation and rendering of complex water surfaces, ACM Trans. Graph. 21 (3) (2002) 736-744.

D. Enright, F. Losasso, R. Fedkiw, A fast and accurate semi-lagrangian particle level set method, Comput. Struct. 83 (6-7) (2005) 479-490.

B. Franklin, W. Brownrigg, Mr. Farish 1774, Phil. Tans. Roy. Soc. London 64: 445-460.

T. Fu, Y. Ma, D. Funfschilling, H. Z. Li, Bubble formation and breakup mechanism in a microfluidic flowfocusing device, Chem. Eng. Sci. 64 (10) (2009) $2392-2400$.

P. Garstecki, H. A. Stone, G. M. Whitesides, Mechanism for flow-rate controlled breakup in confined geometries: A route to monodisperse emulsions, Phys. Rev. Lett. 94 (2005) 164501.

P. Garstecki, M. J. Fuerstman, H. A. Stone, G. M. Whitesides, Formation of droplets and bubbles in a microfluidic T-junction-scaling and mechanism of break-up, Lab Chip 6 (2006) 437-446.

D. Gueyffier, J. Li, A. Nadim, R. Scardovelli, S. Zaleski, Volume-of-fluid interface tracking with smoothed surface stress methods for three-dimensional flows, J. Comput. Phys. 152 (2) (1999) 423-456.

P. Guillot, A. Colin, Stability of parallel flows in a microchannel after a T junction, Phys. Rev. E 72 (2005) 066301.

C. Hirt, B. Nichols, Volume of fluid (VOF) method for the dynamics of free boundaries, J. Comput. Phys. 39 (1981) 201-225.

J. Hua, B. Zhang, J. Lou, Numerical simulation of microdroplet formation in coflowing immiscible liquids, AIChE J. 53 (2007) 2534-2548.

A. Huebner, S. Sharma, M. Srisa-Art, F. Hollfelder, J. B. Edel, A. J. deMello, Microdroplets: A sea of applications? Lab Chip 8 (2008) 1244-1254.

D. Jacqmin, Calculation of two-phase Navier-Stokes flows using phase-field modeling, J. Comput. Phys. 155 (1999) 96-127.

C. Liu, J. Shen, A phase field model for the mixture of two incompressible fluids and its approximation by a fourier-spectral method, Physica D 179 (3-4) (2003) 211-228.

H. H. Liu, Y. H. Zhang, Droplet formation in a T-shaped microfluidic junction, J. Appl. Phys. 106 (2009) 034906.

H. H. Liu, Y. H. Zhang, The mechanism of droplet formation in the squeezing regime in microfluidic crossjunction, (submitted to Phys. Fluids)

T. Nisisako, T. Torii, T. Higuchi, Droplet formation in a microchannel network, Lab Chip 2 (2002) 24-26.

S. Osher, R. P. Fedkiw, Level Set Methods and Dynamic Implicit Surfaces, Springer, 2003.

S. Osher, J. A. Sethian, Fronts propagating with curvature-dependent speed: Algorithms based on hamiltonjacobi formulations, J. Comput. Phys. 79 (1) (1988) 12-49. 
M. Steegmans, C. Schron, R. Boom, Generalised insights in droplet formation at T-junctions through statistical analysis. Chem Eng Sci. 2009; 64(13):3042 - 3050.

S. Sugiura, M. Nakajima, M. Seki, Prediction of droplet diameter for microchannel emulsification: Prediction model for complicated microchannel geometries, Ind. Eng. Chem. Res. 43 (2004) 8233-8238.

M. Sussman, E. Fatemi, An efficient, interface-preserving level set redistancing algorithm and its application to interfacial incompressible fluid flow, SIAM J. Sci. Comput. 20 (4) (1999) 1165-1191.

J. Tan, J. Xu, S. Li, G. Luo, Drop dispenser in a cross-junction microfluidic device: Scaling and mechanism of break-up, Chem. Eng. J. 136 (2008) $306-311$.

T. Thorsen, R.W. Roberts, F. H. Arnold, S. R. Quake, Dynamic pattern formation in a vesicle-generating microfluidic device, Phys. Rev. Lett. 86 (2001) 4163-4166.

J. D. Tice, H. Song, A. D. Lyon, R. F. Ismagilov, Formation of droplets and mixing in multiphase microfluidics at low values of the Reynolds and the Capillary numbers, Langmuir 19 (2003) 9127-9133.

G. Tryggvason, B. Bunner, A. Esmaeeli, D. Juric, N. Al-Rawahi, W. Tauber, J. Han, S. Nas, Y.-J. Jan, A fronttracking method for the computations of multiphase flow, J. Comput. Phys. 169 (2) (2001) 708-759.

P. B. Umbanhowar, V. Prasad, D. A. Weitz, Monodisperse emulsion generation via drop break off in a coflowing stream, Langmuir 16 (2000) 347-351.

S. O. Unverdi, G. Tryggvason, A Front Tracking Method for Viscous Incompressible Flows. J. Comput. Phys. 100 (1992) 25-37.

S. van der Graaf, T. Nisisako, C. G. P. H. Schroën, R. G. M. van der Sman, R. M. Boom, Lattice Boltzmann simulations of droplet formation in a T-shaped microchannel, Langmuir 22 (2006) 4144-4152.

S. van der Graaf, T. Nisisako, C. G. P. H. Schroën, R. G. M. van der Sman, R. M. Boom, Lattice Boltzmann simulations of droplet formation in a T-shaped microchannel, Langmuir 22 (2006) 4144-4152.

J. Xu, S. Li, J. Tan, G. Luo, Correlations of droplet formation in Tjunction microfluidic devices: from squeezing to dripping, Microfluid Nanofluid 5 (2008) 711-717.

T. Yabe, F. Xiao, Description of Complex and Sharp Interface with Fixed Grids Incompressible and Compressible Fluid, Computers Math. Applic 29 (1995).

M. Yasuno, S. Sugiura, S. Iwamoto, M. Nakajima, A. Shono, K. Satoh, Monodispersed microbubble formation using microchannel technique, AIChE J. 50 (2004) 3227-3233.

B. Zheng, J. D. Tice, L. S. Roach, R. F. Ismagilov, A droplet-based, composite PDMS/glass capillary microfluidic system for evaluating protein crystallization conditions by microbatch and vapor-diffusion methods with onchip x-ray diffraction, Angew. Chem. Int. Ed. 43 (2004) 2508-2511. 